
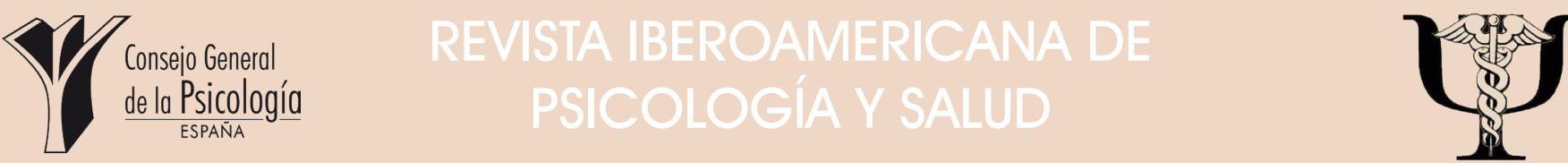

Revista Oficial de la Federación Iberoamericana de Asociaciones de Psicología (FIAP)

[Official Journal of the Latin-American Federation of Psychological Associations]

\title{
Personalidad y gravedad delictiva en adolescentes con conducta antisocial persistente
}

Paula A. Alarcón*, Ricardo X. Pérez-Luco, Lorena S. Wenger, Sonia I. Salvo y Sergio

\author{
A. Chesta \\ Universidad de La Frontera
}

- Recibido: 6-11-2017 - Aceptado: 5-12-2017 - Avance online: 18 - 12 - 2017

RESUMEN: Enmarcado en los desarrollos teóricos de la criminología evolutiva, el objetivo de este estudio fue reconocer patrones de personalidad que comandan agrupaciones diferenciadas de adolescentes infractores persistentes, determinando la relación entre variables de personalidad y gravedad delictiva. Participaron en el estudio 330 adolescentes varones con sanción penal en Chile, evaluados con una bateria multidimensional de 5 instrumentos. Las variables personales, medidas con $\mathrm{MACl}$, se utilizan para establecer los clúster usando la técnica de análisis Biplot, las restantes mediciones (conducta antisocial, riesgos, recursos y salud mental) se han usado para caracterizar los grupos emergentes y compararlos entre sí. Los resultados identifican 5 clústers diferenciados cuyos perfiles de personalidad se interpretan y se etiquetán con las iniciales $A E, E A, P D, T V$ y $N D$ que reconocen las variables dominantes en cada caso; luego se comparan entre sí, estableciendo una gradiente de gravedad delictiva que resulta consistente y donde las variables de personalidad juegan un rol activo en la génesis y mantención de la conducta o como factor que favorece el desistimiento. Se discuten los resultados en función de su pertinencia para la implementación de programas de intervención efectivos, así como sus implicaciones para la reducción y prevención del delito.

PALABRAS CLAVE: MACI, Delincuencia juvenil, Riesgo criminógeno, Salud mental, Desistimiento.

\section{Personality and offense severity in adolescents with persistent antisocial behavior}

ABSTRACT: The objective of this study was to recognize patterns of personality that command differentiated groups of persistent offenders, determining the relationship between personality variables and the severity of criminal behavior. We studied a sample of 330 male adolescents sanctioned in Chile, evaluated with a multidimensional battery of 5 instruments. Personal variables, measured with $\mathrm{MACl}$, are used to establish clusters using the Biplot analysis technique, the remaining measurements (criminal behavior, risks, resources and mental health) are used to characterize emerging groups and compare them to each other. Five distinct clusters are obtained, whose personality profiles are interpreted, labeling them with the initials $A E, E A, P D, T V$ and ND that recognize the dominant variables in each case; Then they compare each other, establishing a gradient of criminal gravity that is very consistent and where personality variables play an active role in the genesis and maintenance of behavior or as a factor that favors desistance. The results are discussed according to their relevance to the implementation of effective and relevant intervention programs in crime reduction and prevention.

KEYWORDS: MACl, Young offenders, Criminogenic risk, Mental health, Desistance.

Las conductas agresivas y antisociales, expresadas con más frecuencia en la adolescencia, refieren un proceso de desajuste personal y social multidimensional que ha generado vasta evidencia para comprender el inicio, mantención y desistimiento delictivo en el curso de la vida. La alta concentración

*Correspondencia: Paula Alarcón

Departamento de Psicología, Universidad de La Frontera. AV.

Código Postal: 01145 , Av. Francisco Salazar, Temuco, Chile

E-mail:paula.alarcon@ufrontera.cl

(C) 2018 Sociedad Universitaria de Investigación en Psicología y Salud. Publicado por Consejo General de Colegios Oficiales de Psicólogos, España. Este es un artículo Open Access
bajo la CC BY-NC-ND licencia (http://creativecommons.org/licencias/by-nc-nd/4.0/). de comportamientos antisociales durante la adolescencia ha sido un indicador reiterado en diferentes estudios de seguimiento (Moffitt, 1993, 2003; Piquero, Farrington, Nagin, \& Moffitt, 2010), alcanzando cifras que podrían homologarse a una desviación normativa del desarrollo (Bosick, Bersani, \& Farrington, 2015). No obstante, la misma evidencia demuestra que es un grupo minoritario el que persiste en comportamientos antisociales en la adultez, siendo foco de mayor preocupación la complejizacion de la conducta antisocial que eleva su productividad, versatilidad y 
gravedad delictiva (Pérez-Luco et al., 2017). Esta delincuencia persistente (Moffitt, 2003) acumularía más factores de riesgo criminógeno (Andrews, Bonta \& Wormith, 2011 ; RodríguezDíaz et al., 2011; Piquero, Jennings \& Barnes, 2012), junto a variables personales predisponentes (Fréchette \& Le Blanc, 1998), siendo esta coexistencia determinante de la mantención de los comportamientos antisociales (Bosick et al., 2015).

La evidencia actual muestra la eficacia de intervenciones basadas en el modelo RNR que focaliza factores de riesgo dinámicos, como desenganche escolar progresivo, abuso de drogas y alcohol (Guillén, Roth, Alfaro, \& Fernández, 2015), amigos con alto compromiso delictivo, (Alarcón, Pérez-Luco, Chesta, \& Wenger, 2017; Olver, Stockdale, \& Wormith, 2014); y su impacto se eleva cuando la intervención se ajusta a variables personales, como lo propone el MIID (Fréchette \& Le Blanc, 1998).

En criminología se reconocen al menos tres líneas de investigación con evidencia que conecta rasgos de personalidad y comportamiento antisocial. La primera que Morizot (2015) describe como (i) predisposición, plantea que los rasgos de personalidad tienen un efecto en el inicio del comportamiento delictivo (Jolliffe \& Farrington, 2009; Moffitt, 2015; Tremblay, Côté, Salla, \& Michel, 2017); la segunda, (ii) plasticidad, señala a la personalidad como un factor de activación, pero también de agravamiento del comportamiento antisocial (De Bolle, Beyers, De Clercq, \& De Fruyt, 2012; Fréchette \& Le Blanc, 1998; Le Corff \& Toupin, 2010); y la tercera (iii) de remisión (Le Blanc \& Loeber, 1998) que enfatiza el efecto de la personalidad en procesos de desistimiento del comportamiento delictivo. Además, se ha encontrado en un metanálisis reciente (Olver, Stockdale \& Wormith, 2014), que la tendencia personal antisocial, asumida en el modelo RNR, es uno de los 6 factores de riesgo de mayor tamaño de efecto en la predicción de reincidencia. Esta evidencia la respaldan estudios de seguimiento que coinciden al señalar que las variables personales juegan un rol diferenciador de trayectorias delictivas persistentes (Farrington,
Ttofi, \& Piquero, 2016; Fréchette \& Le Blanc, 1998; Loeber et al., 2003). La personalidad sería una variable relevante, pero insuficiente por sí sola, para explicar la persistencia de la delincuencia en jóvenes (Alarcón, Pérez-Luco, \& Wenger, 2017).

Al aislar variables específicas que intervienen en comportamientos antisociales en la niñez y adolescencia, la impulsividad destaca por la mayor evidencia acumulada a través del tiempo (Higgins, Kirchner, Ricketts, \& Marcum, 2013; Siödin, Wallinius, Billstedt, Hofvander, \& Nilsson, 2017). Se demuestra la evolución de la relación diádica entre hiperactividad-impulsividad y las conductas antisociales persistentes en la adultez (Jolliffe \& Farrington, 2009; Moffitt et al., 2011); y en el grupo de inicio precoz resaltan déficits en patrones de crianza familiar y presencia de variables neuropsicológicas que afectarían la interpretación de las experiencias (Moffitt, 2015).

También destacan insensibilidad social, baja empatía y un déficit afectivo, como precusores de personalidad antisocial (Frick \& Dickens, 2006), y se ha investigado el constructo de psicopatía. Para Hare (2003), psicopatía contempla cuatro facetas; dos en un nivel afectivo e interpersonal y dos referidas a estilo de vida y conducta criminal; recientemente Pérez, Herrero, Velasco, \& Rodríguez-Díaz, (2015), aportan evidencia de validez de contructo para 3 factores en el PCL-R, dejando fuera los ítems de conducta criminal; se discute que esta alteración en la afectividad no necesariamente tendría su expresión en todos los casos en comportamiento delictivo (Pérez, Rodríguez-Díaz, Herrero, \& Fernández-Suárez, 2016). La personalidad antisocial se señala como un patrón independiente de los otros con un estilo de afrontamiento activo y autónomo frente al entorno, baja ansiedad, desconfianza hacia los demás, y vulneración de los derechos de los otros, pudiendo mantenerse en el límite de lo socialmente permitido (Millon, Simonsen, Birket-Smith, \& Davis, 2003).

También se ha estudiado la relación entre capacidad de vinculación afectiva, apego, y delincuencia, encontrando asociación entre apego familiar, procesos de desajuste 
emocional y trastornos de adaptación social (García, Moral, Frías, Valdivia, \& Díaz, 2012; Gómez, Arango, Molina, \& Barceló, 2010). Estudios recientes aportan evidencia de interacción de procesos cognitivos y emocionales en la predicción de la conducta agresiva y prosocial en adolescentes con y sin comportamiento delictivo (Llorca-Mestre, Malonda-Vidal, \& Samper-García, 2017); y de los efectos mediadores de las prácticas parentales y la relación con pares desviados en el comportamiento desadaptativo durante la adolescencia (Cutrín, Gómez-Fraguela, Maneiro, \& Sobral, 2017); en ambos casos se observan efectos diferenciados de las variables predictoras, siendo más probable la conducta agresiva o violenta cuando hay baja empatía, mayor impulsividad, menor toma de perspectiva y mayor influencia de pares desviados. La mayor visibilización de las competencias personales en el plano cognitivo-comportamental y de personalidad, asociadas a la persistencia y gravedad delictiva, fue demostrado por Fariña, Vázquez y Arce (2014) y se ha encontrado en metanálisis recientes (Assink et al., 2015).

En síntesis, la evidencia permite afirmar la existencia de dos dimensiones: (i) menor autoregulación de la conducta, asociada a impulsividad-hiperactividad, ausencia de ansiedad, inestabilidad emocional y un estilo buscador de emociones; y (ii) menor resonancia emocional y vinculación afectiva con los otros, (Barlett \& Anderson, 2012; Bergheul \& Collette, 2013; Jones, Miller, \& Lynam, 2011; Le Corff \& Toupin, 2009). En consecuencia, se adopta una mirada multidimensional, enfatizando el rol dinámico de la personalidad en la activación, agravamiento y desistimiento del comportamiento antisocial.

Para este estudio, la teoría evolutiva de Theodore Millon (1990) ofrece un andamiaje sinérgico al integrarla con la medición de variables del entorno, definiendo personalidad como: "...patrón complejo de características psicológicas profundamente arraigadas, (...) en su mayor parte inconscientes y difíciles de cambiar, (que) se expresan automáticamente en casi todas las áreas del funcionamiento (...)". Estos rasgos intrínsecos y generales surgen de una complicada matriz de determinantes biológicos y aprendizajes, comprenden el modo idiosincrático de percibir, sentir, pensar, afrontar y comportarse de un individuo" (Millon \& Davis, 1998, p. 4).

El Inventario MACl (Millon, 1993) aporta una buena medición del funcionamiento psicológico y conductual de los adolescentes (Romm, Bockian, \& Harvey, 1999) junto con la diferenciación de trastornos específicos (Faúndez \& Vinet, 2009; Ferrer \& Kirchner, 2014), permitiendo discriminar características de adolescentes infractores de ley, donde las escalas transgresor y poderoso, junto a insensibilidad social, predisposición delictual, tendencia al abuso de substancias, impulsividad $y$ ansiedad muestran el mayor tamaño de efecto al diferenciar a este grupo de adolescentes de una muestra equivalente, pero convencional.

Murrie y Cornell (2000), explorando la capacidad del $\mathrm{MACl}$ para evaluar tendencia antisocial en jóvenes privados de libertad, encuentran correlaciones significativas con las escalas transgresor y tendencia al abuso de sustancias; derivando dos subescalas de psicopatía, una de 20 ítemes (Murrie \& Cornell, 2000) y otra de 16 (Salekin, Ziegler, Larrea, Anthony, \& Bennett, 2003). Con el PCL:YV (Forth, Kosson, \& Hare, 2003) se ha obtenido en jóvenes correlaciones entre las facetas de psicopatía y las escalas transgresor, poderoso y oposicionista (Zúñiga, Vinet, \& León, 2011). También se ha descrito la personalidad de adolescentes mujeres ofensoras de ley (Stefurak \& Calhoun, 2007), adolescentes que ejercen violencia filio-parental (Castañeda, Garrido-Fernández, \& Lanzarote, 2012) y con conductas violentas (Kennedy, Burnett, \& Edmonds, 2011); y abusadores sexuales juveniles (Glowacz \& Born, 2013; Morrell \& Burton, 2014). El análisis de clúster es la técnica de uso más frecuente para agrupar variables de personalidad diferenciadoras en jóvenes con conductas delictivas (Bosick, Bersani, \& Farrington, 2015; Stefurak, Calhoun, \& Glaser, 2004; Taylor, Kemper, Loney \& Kistner, 2006; Wareham, Dembo, Poythress, Childs \& Schmeidler; 2009), observándose que la mayoria de los estudios reconocen entre 4 y 5 clúster, destacando un estilo transgresor, impulsividad, el componente de ansiedad y características de un patrón antisocial. 
Con esta evidencia, el problema a resolver es si el análisis de variables personales en adolescentes con delincuencia persistente permite determinar agrupaciones diferenciadas en Chile. Así, se define como objetivo de estudio determinar grupos de adolescentes con distintas configuraciones de patrones de personalidad, y caracterizarlos comparativamente según gravedad delictiva, factores de riesgo criminógeno, recursos de adaptación y salud mental.

\section{MÉTODO}

\section{- PARTICIPANTES}

La población del estudio corresponde a los adolescentes varones sancionados penalmente durante el año 2011 en cuatro regiones del sur de Chile. De esta población $(n=2.185)$ se obtuvo una muestra incidental de 571 participantes, de la cual se extrae la muestra de estudio, usando los siguientes criterios: (a) ser hombre, (b) menor a 20 años; (c) tener una sanción mayor a seis meses, (d) presentar delincuencia persistente, y (e) consentir su participación. La muestra completa consintió su participación, de ellos, 60 son mujeres, 50 corresponden a delincuencia transitoria, 21 a delincuencia compleja y 440 a delincuencia persistente; los indicadores para establecer un patrón delictivo persistente fueron edad de inicio precoz (menor o igual a 13 años), agravamiento delictivo, 13 o más delitos autoreportados, y tres o mas judicializaciones (Pérez-Luco et al., 2017).

De los 440 jóvenes que se tipifican con delincuencia persistente, 22 tienen sanciones inferiores a 6 meses, 4 son mayores de 20 años, 63 no responden $\mathrm{MACl}$ y 21 lo invalidan, por lo que se trabajó con 330 jóvenes que cumplían los 5 criterios. Esta muestra, al momento de ser evaluada tenía edad promedio de 17.2 años, inicio delictivo a los 11.2; estudios básicos completos ( $M=8.1$ años), una media de 364 delitos auto-informados, 3.4 judicializados $(<1 \%) ;$ y 2.3 años de condena con $25 \%$ ya cumplida ( $M=7$ meses). Un 67\% cumplía sanción en medio libre y $33 \%$ se encontraba privado de libertad.

\section{- INSTRUMENTOS DE MEDIDA}

\section{INVENTARIO CLÍNICO PARA ADOLESCENTES} DE MILLON, MACI (Millon, 1993). Cuestionario de autoinforme de construcción racional, se compone de 160 ítems ( $V$ o F) que originan 33 escalas distribuídas en cinco áreas (patrones de personalidad -12-, preocupaciones adolescentes -8-, síndromes clínicos -7-; contenido psicopático -2-; y validez -4-). En este estudio se usó la versión chilena (Alarcón, Vinet, \& Salvo, 2005; Vinet \& Forns, 2008), en formato adaptado para adolescentes infractores, que evidencia características psicométricas aceptables para su uso (valores $\alpha$ entre .72 y .88; en las escalas analizadas; con $n=658)$.

\section{ESCALA DE DELINCUENCIA AUTORREVELADA,} EDA (Pérez-Luco, Lagos, Chesta, \& Báez, 2014). Formulario de entrevista guiada que se aplica en la intervención con adolescentes, permitiendo indagar en profundidad en sus formas de actuación delictiva. Tiene 63 ítems de consulta sobre diferentes tipos de delitos, organizados en tres secciones: (a) hurtos y robos, (b) agresiones y (c) otros delitos; en cada ítem se consulta frecuencia de realización en siete rangos de edad ( $<8$ años a 19 o más). Su consistencia interna $(\alpha)$ es de .95 para hurtos y robos, .80 para agresiones y .61 para otros delitos. Su valor radica en identificar patrones delictivos persistentes, especializados y polimórficos.

\section{ESCALA DE GRAVEDAD DEL ENGANCHE} DELICTIVO, EGED (Lagos, Pérez-Luco, Chesta, \& Wenger, 2014). Inventario de juicio profesional estructurado que determina el grado de adhesión a un patrón delictivo persistente, diferenciándolo de delincuencia transitoria y con base en evidencia empírica chilena. Consta de 12 ítemes dicotómicos (presencia-ausencia) que califican los profesionales previa revisión de antecedentes y realización de entrevistas al adolescente e informantes calificados. Un puntaje $\geq 3$ es indicativo de delincuencia persistente, pero también lo son por sí solos 4 de los 12 indicadores. Se inspira en la guía usada en los Centres de Jeunesse de Montréal, 
Canadá, pero su desarrollo y validación es con adolescentes chilenos, mostrando alta consistencia interna con la muestra de estudio $(\alpha=.81 ; n=384)$.

\section{FICHA DE EVALUACIÓN DE RIESGOS Y} RECURSOS, FER-R (Alarcón, Wenger, Chesta, \& Salvo, 2012). Inventario de juicio profesional estructurado de 57 ítems, que permite el registro de: (a) riesgos criminógenos (intervenciones previas, educación, pares, familia; drogas, y actitudes); y (b) recursos para la intervención (recursos personales y familiares e intereses). Genera una puntuación total de riesgo de reincidencia (0-39 pts.), que orienta sobre la intensidad de la intervención; ofrece un perfil de riesgos específicos asociados a puntuaciones parciales por factor que permite focalizar las acciones, además de una puntuación total de factores protectores (0-18 pts.) que permite ajustar el plan de intervención a las capacidades del joven. Se encuentra validada y estandarizada en Chile (Alarcón et al., 2012), mostrando diversas evidencias de validez (convergente, acuerdo interjueces y predictiva) e índices de consistencia interna altos en la muestra de estudio $(n=392)$ para las escalas principales, riesgos criminógenos ( $\alpha=.93)$ y recursos para la intervención $(\alpha=.86)$.

\section{CUESTIONARIO DE EXPLORACIÓN DE SALUD MENTAL PARA ADOLESCENTES, CESMA (Berríos} et al., 2014). Formulario de entrevista guiada para tamizaje de posibles dificultades de salud mental en las áreas de mayor prevalencia de trastornos en la adolescencia. Inspirado en el SQIFA (Protocolo ASSET de Inglaterra); está validado para adolescentes infractores chilenos, revelando alta consistencia interna en la muestra de estudio ( $\alpha=.82 ; n=392)$. Se estructura en tres secciones: síntomas, tratamientos y cuadros clínicos; la primera se subdivide en 6 factores (alcohol, drogas, ansiedad, depresión, estrés postraumático y autoagresiones) con puntaje de 0-4 en cada uno (ausencia a recurrencia).

\section{- PROCEDIMIENTO}

Los adolescentes fueron evaluados con el protocolo del proyecto FONDEF D08i-1205
(Pérez-Luco, Zambrano, Alarcón, \& Alarcón, 2014) por 48 profesionales con responsabilidad en sus sanciones, previa especialización de 584 hrs. y firma de un compromiso de confidencialidad. A los jóvenes se les informó del carácter del estudio y se les solicitó consentimiento antes de sus respuestas y todo el proceso se realizó en el marco de convenios institucionales formales.

\section{- ANÁLISIS}

Se usó un análisis de conglomerado jerárquico de las coordenadas obtenidas con el Biplot simétrico de Gabriel (2002), y la técnica gráfica multidimensional Biplot PMD (MartinsVairinhos \& Galindo, 2004), por la calidad de representación de las variables involucradas a través de la longitud de sus vectores (escalas de personalidad); los vectores más largos (variables eje) muestran mayor varianza intersujetos, delimitando clúster diferenciados y permitiendo describir mejor al grupo con el que se asocian en el plano. Este análisis se hace con las 12 variables de patrones de personalidad del $\mathrm{MACl}$, para luego conceptualizar los conglomerados obtenidos con base en la interpretación de perfiles promedio de cada grupo (incluidas las otras variables MACI).

Para establecer la variación de los patrones entre clúster se realizó un ANOVA y se comparó y caracterizó las agrupaciones con las medidas obtenidas en las restantes variables (comportamiento, riesgos, recursos y salud mental), usando la prueba no paramétrica de Kruskal-Wallis debido a las distribuciones asintóticas de los datos. Finalmente se estimaron los tamaños de efecto con la prueba $G$ de Hedges para las comparaciones con diferencias estadísticamente significativas (Lenhard \& Lenhard, 2016).

\section{RESULTADOS}

\section{- DETERMINACIÓN DE CLÚSTER}

El análisis diferencia cinco clúster. En el centro se forma un grupo con puntuaciones bajas en todas las escalas, y de allí en todas direcciones se forman otros cuatro clúster; cada 


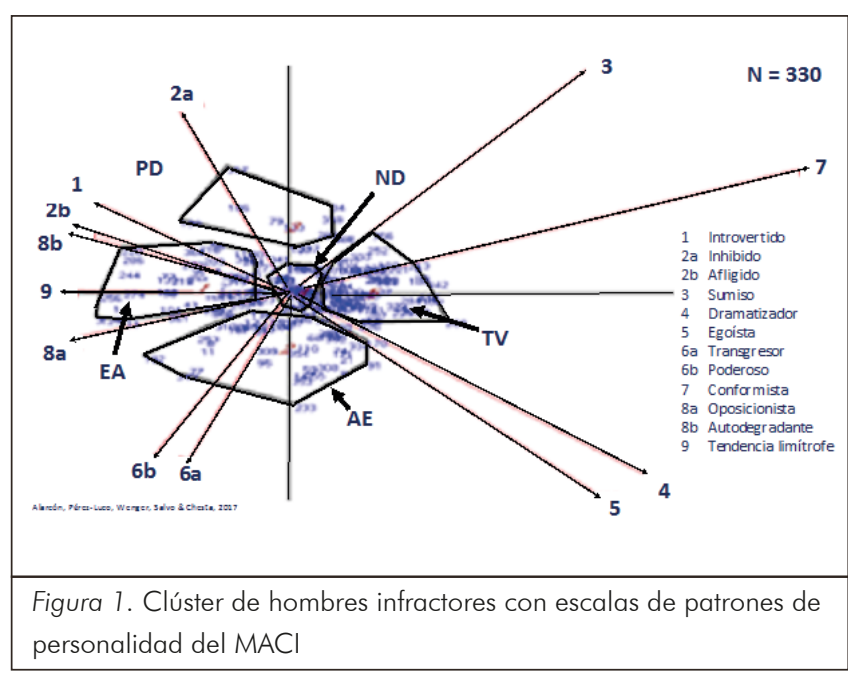

uno con una constelación diferente de variables dominantes y en oposición a otras (ver figura 1). Constituidos los grupos y usando ANOVA, con las 29 variables de $\mathrm{MACl}$, se detectan 6 diferenciadoras que permiten decribir psicológicamente cada grupo, nombrándolos: antisocial estabilizado "AE", explosivo autodestructivo "EA", pasivo desesperanzado "PD", transgresor vinculado "TV" y normal desviado "ND"; estos se caracterizan posteriormente con las variables descriptivas de gravedad delictiva, riesgos criminógenos, recursos de adaptación y salud mental.

Las escalas transgresor (6a; $\alpha=.82)$, poderoso (6b; $\alpha=.77)$, insensibilidad social $(F ; \alpha=.72)$, tendencia a la impulsividad (DD; $\alpha=.72$ ), sentimientos ansiosos (EE; $\alpha=.81$ ) y psicopatía (MC20; $\alpha=.76$ ), muestran diferencias estadísticamente significativas entre los cinco clúster, con tamaños de efecto considerables en 56 de las 60 comparaciones realizadas (ver tabla 1), lo que transformado a puntaje estándar revela una gradación decreciente entre los grupos al comparar sus puntajes promedio estandarizados (percentiles) en cada variable; si se asume que la población normal se ubica entre 30 y 70 puntos, se puede observar la gradiente del peso de las variables personales en la determinación de

Tabla 1

Comparación de medias en variables de personalidad, diferenciadoras de clúster

\begin{tabular}{|c|c|c|c|c|c|c|c|c|c|}
\hline \multirow{2}{*}{\multicolumn{3}{|c|}{ Variables y Clúster }} & \multicolumn{3}{|c|}{ Índice $G$ de Hedges } & \multicolumn{4}{|c|}{ ANOVA } \\
\hline & & & \multirow{2}{*}{$\begin{array}{l}\mathrm{AE} \\
.96\end{array}$} & \multirow[t]{2}{*}{ EA } & \multirow[t]{2}{*}{ PD } & \multirow[t]{2}{*}{ TV } & \multirow[t]{2}{*}{ gl total } & \multirow[t]{2}{*}{$F$} & \multirow[t]{2}{*}{$p$} \\
\hline \multirow{24}{*}{$\begin{array}{l}\text { Escalas } \\
\mathrm{MACl}\end{array}$} & \multirow{4}{*}{ Transgresor } & EA & & & & & & & \\
\hline & & PD & 1.85 & .53 & & & \multirow{3}{*}{329} & \multirow{3}{*}{206.5} & \multirow{3}{*}{.000} \\
\hline & & TV & 2.88 & 1.39 & .95 & & & & \\
\hline & & ND & 5.63 & 3.25 & 3.37 & 2.49 & & & \\
\hline & \multirow{4}{*}{ Poderoso } & EA & .14 & & & & \multirow{4}{*}{329} & \multirow{4}{*}{97.3} & \multirow{4}{*}{.000} \\
\hline & & PD & 1.20 & .82 & & & & & \\
\hline & & TV & 2.20 & 1.67 & .85 & & & & \\
\hline & & ND & 3.39 & 2.47 & 2.14 & 1.57 & & & \\
\hline & \multirow{4}{*}{$\begin{array}{c}\text { Insensibilidad } \\
\text { Social }\end{array}$} & EA & 1.63 & & & & \multirow{4}{*}{329} & \multirow{4}{*}{70.5} & \multirow{4}{*}{.000} \\
\hline & & PD & 1.90 & .10 & & & & & \\
\hline & & TV & 1.58 & .46 & .19 & & & & \\
\hline & & ND & 2.69 & .64 & 1.03 & 1.54 & & & \\
\hline & \multirow{4}{*}{$\begin{array}{c}\text { Tendencia } \\
\text { a la } \\
\text { Impulsividad }\end{array}$} & EA & .85 & & & & \multirow{4}{*}{329} & \multirow{4}{*}{137.5} & \multirow{4}{*}{.000} \\
\hline & & PD & 1.55 & .55 & & & & & \\
\hline & & TV & 2.15 & 1.16 & .65 & & & & \\
\hline & & ND & 5.04 & 3.26 & 2.78 & 1.82 & & & \\
\hline & \multirow{4}{*}{$\begin{array}{l}\text { Sentimientos } \\
\text { ansiosos }\end{array}$} & EA & 1.84 & & & & \multirow{4}{*}{329} & \multirow{4}{*}{76.4} & \multirow{4}{*}{.000} \\
\hline & & PD & 1.75 & .27 & & & & & \\
\hline & & TV & 1.54 & .62 & .34 & & & & \\
\hline & & ND & 3.31 & .82 & 1.32 & 1.85 & & & \\
\hline & & EA & .16 & & & & & & \\
\hline & Psicopatía & PD & 1.41 & 1.12 & & & 320 & 1210 & 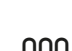 \\
\hline & Cornell & TV & 2.12 & 1.80 & .74 & & 209 & 124.9 & .000 \\
\hline & & ND & 3.66 & 3.13 & 2.47 & 1.68 & & & \\
\hline
\end{tabular}


Tabla 2

Puntaje estándar promedio en variables de personalidad diferenciadoras de clúster

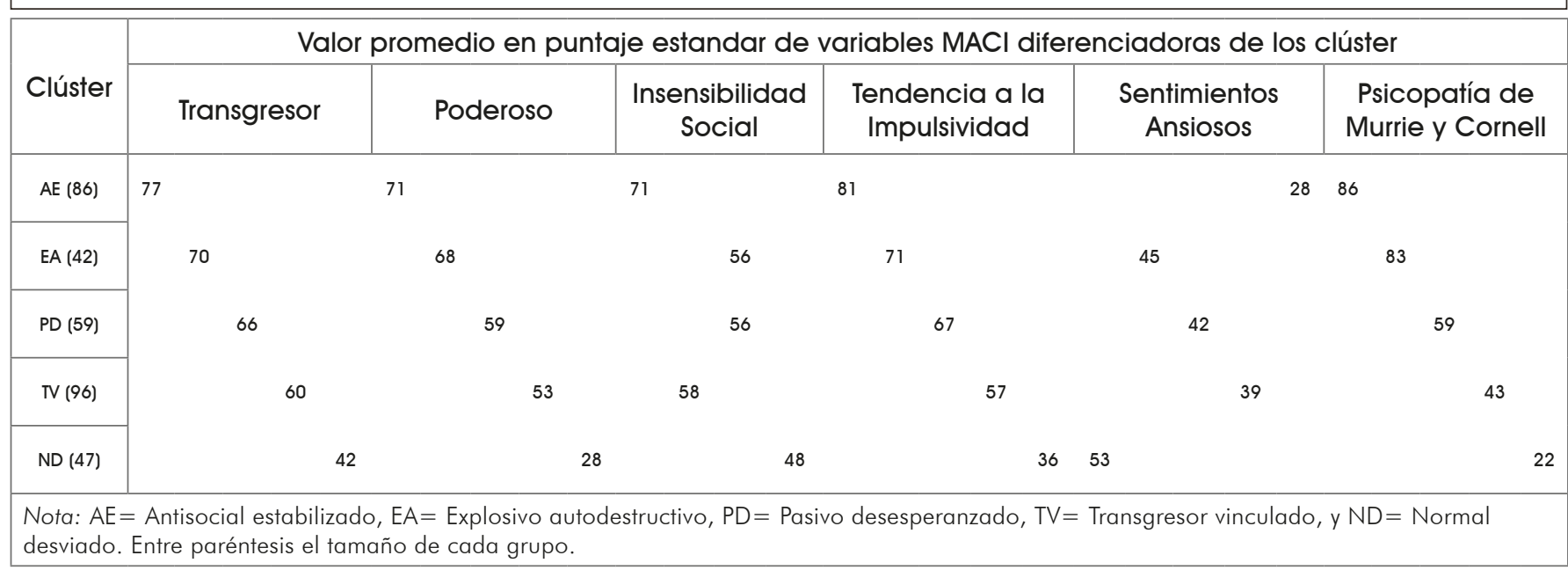

la conducta delictiva de cada clúster, pues los puntajes se ordenan de mayor a menor, salvo en sentimientos ansiosos, que su peso es inverso -baja ansiedad- (ver tabla 2).

\section{- CARACTERIZACIÓN PSICOLÓGICA DE LOS CLÚSTER}

Usando las medianas de las 29 escalas del $\mathrm{MACl}$, se dibuja para cada clúster un perfil tipo que se interpreta con base en las puntuaciones de las escalas dominantes, de acuerdo a las directrices del modelo teórico de Millon, derivando una caracterización psicológica por grupo con las variables más acentuadas.

\section{AE (ANTISOCIAL ESTABILIZADO, 26\%).}

Dominan el perfil transgresor (6a) y poderoso (6b), revelando un estilo activo, autónomo y manipulador ante el entorno, con exacerbada orientación a la satisfacción de necesidades personales y probable comportamiento abusivo hacia los otros. Presentan seguridad en si mismo, e insensibilidad social (F), con marcada impulsividad (DD), tendencia al consumo de drogas (BB), predisposición a delinquir (CC) y elevada puntuación en psicopatía (MC20). La personalidad sería un factor de riesgo, por la especial orientación hacia si mismo, escasa capacidad de vinculación afectiva, reducida preocupacion por el impacto de sus acciones en su comunidad y alta insensibilidad social que valida el comportamiento delictivo.
EA (Explosivo Autodestructivo, 13\%). Se elevan los patrones afligido (2b), transgresor (6a) poderoso (6b) autodegradante (8b) y tendencia limítrofe (9). Esto implica inestabilidad y desorganización emocional, incurriendo en comportamientos impulsivos con mayor compromiso de violencia asociada a emocionalidad no regulada. Sus acciones tienden a ser poco planificadas, pero muestra alta receptividad, buscando la atención afectiva de los otros. La personalidad en este grupo se postula en el inicio, y especialmente en el agravamiento de los comportamientos delictivos mediados por su impulsividad, donde las agresiones y la violencia acompañan todo su actuar, incluyendo autoagresiones y escasa regulación emocional.

PD (Pasivo Desesperanzado, 18\%). Comandada por las escalas afligido (2b), inhibido (2a) introvertido (1), autodegradante (8b) y oposicionista (8a); se caracterizan por dificultad para experimentar placer y anticipar el dolor psícológico, percibiendo la vida de modo pesimista con escasa esperanza de recompensas. Muestra especial tendencia a inhibir sus emociones, exhibiendo hacia el entorno sentimientos de desgano, desmotivación, aburrimiento y hostilidad. El proceso de desarrollo adolescente se muestra con conflictos de identidad y desvalorización de sí mismos, reportando experiencias de maltrato y daño en la infancia y revelando alta tendencia al abuso de sustancias y alcohol, con muy baja autoestima y predominio de un estilo pasivo para afrontar las experiencias. 
TV (Transgresor Vinculado 29\%). Lo define una leve elevación de la escala transgresor (6a), por sobre la mediana del grupo 160 puntos,) acompañada de las escalas sumiso (3) y conformista (7), que se interpretan de modo inverso. Revela personalidad rebelde, desafiante y contestataria, que suele saltarse las reglas sociales, pero evitando transgredir los límites de los otros, pues privilegia las relaciones interpersonales sintiendo fuerte necesidad de vincularse. Se diferencian del AE en las escalas poderoso y psicopatía, con puntuación que se ubica dentro de valores normativos, mostrando reconocimiento e interés por el grupo social al que pertenece; empero, las barreras de ansiedad o miedo no sirven como freno o límite a su comportamiento, actuando de modo planificado y justificando su actuar delictivo por las necesidades de su grupo de referencia. En esta agrupación las variables de personalidad actuarían como factor o recurso de protección que reduce el impacto de los factores de riesgo criminógeno mostrando, delitos de baja violencia y con una menor gravedad delictiva.

ND (Normal Desviado, 14\%). Este grupo no muestra ninguna elevación distintiva en patrones de personalidad; exhibiendo un patrón de un funcionamiento psicológico flexible y ajustado a las demandas del entorno, similar al de adolescentes convencionales. En su perfil se elevan levemente la tendencia a transgredir las normas legales e inmadurez, privilegiando sus intereses y necesidades por sobre las de otros, pero siempre en rangos normales. En este grupo la personalidad no actúa como factor de riesgo, al contrario, se suma a los recursos de adaptación, neutralizando su conducta antisocial que se inicia en la adolescencia y de forma reactiva al entorno, pues se exacerba cuando se asocia a mayores riesgos contextuales. Muestra recursos personales suficientes para desistir del actuar delictivo cuando los riesgos disminuyen.

\section{COMPARACIÓN DE CLÚSTER}

La caracterización sociodemográfica y delictiva de los clúster no evidencia diferencias importantes (ver tabla 3), sólo las hay en años de estudio $(F=4,7 ; p=.001)$, con tamaños de efecto moderdos ( $G=.55$ a .64) y pequeños ( $G=.21$ a .35). En edad de inicio delictivo no hay diferencias entre los 5 clúster $(F=1,4 ; p=.221)$, pero si entre ND y los otros cuatro, con tamaños de efecto pequeños ( $G=.40$ a .45), evidenciando menor peso de uno de los riesgos estáticos más importantes en la predicción de reincidencia (ver figura 2 ). Empero, lo más relevante es que no hay diferencias significativas en en el número de sanciones previas, la duración de la sanción actual y la proporción de privación de libertad, cuando en al autorreporte si las hay en el volumen de delitos y la gravedad de éstos.

Tabla 3

Caracterización de clúster según variables sociodemográficas y delictivas

\begin{tabular}{|c|c|c|c|c|c|c|c|c|c|}
\hline Clúster & $N$ & $\begin{array}{c}\text { Estudios } \\
\text { Aprobados }\end{array}$ & $\begin{array}{c}\text { Edad } \\
\text { Inicio } \\
\text { delictivo }\end{array}$ & $\begin{array}{l}\text { Edad de } \\
\text { Sanción }\end{array}$ & $\begin{array}{l}\text { Duración } \\
\text { de } \\
\text { Sanción }\end{array}$ & $\begin{array}{c}\text { Delitos } \\
\text { Reportados }\end{array}$ & $\begin{array}{c}\text { Delitos } \\
\text { Sancionados }\end{array}$ & $\begin{array}{l}\text { Privación } \\
\text { Libertad }\end{array}$ & $\begin{array}{l}\text { Sancionados } \\
\text { Medio Libre }\end{array}$ \\
\hline $\mathrm{AE}$ & 86 & 8.5 & 10.9 & 16.5 & 26 & 482 & 3.2 & $29 \%$ & $71 \%$ \\
\hline EA & 42 & 7.8 & 11.0 & 16.7 & 32 & 424 & 3.2 & $50 \%$ & $50 \%$ \\
\hline PD & 59 & 7.1 & 11.1 & 16.9 & 26 & 330 & 3.6 & $34 \%$ & $66 \%$ \\
\hline TV & 96 & 8.4 & 11.2 & 16.7 & 30 & 314 & 4.2 & $29 \%$ & $71 \%$ \\
\hline ND & 47 & 8.2 & 12.3 & 16.4 & 27 & 116 & 2.4 & $30 \%$ & $70 \%$ \\
\hline$\Sigma / \mu$ & 330 & 8.1 & 11.2 & 16.7 & 28 & 346 & 3.4 & $33 \%$ & $67 \%$ \\
\hline
\end{tabular}




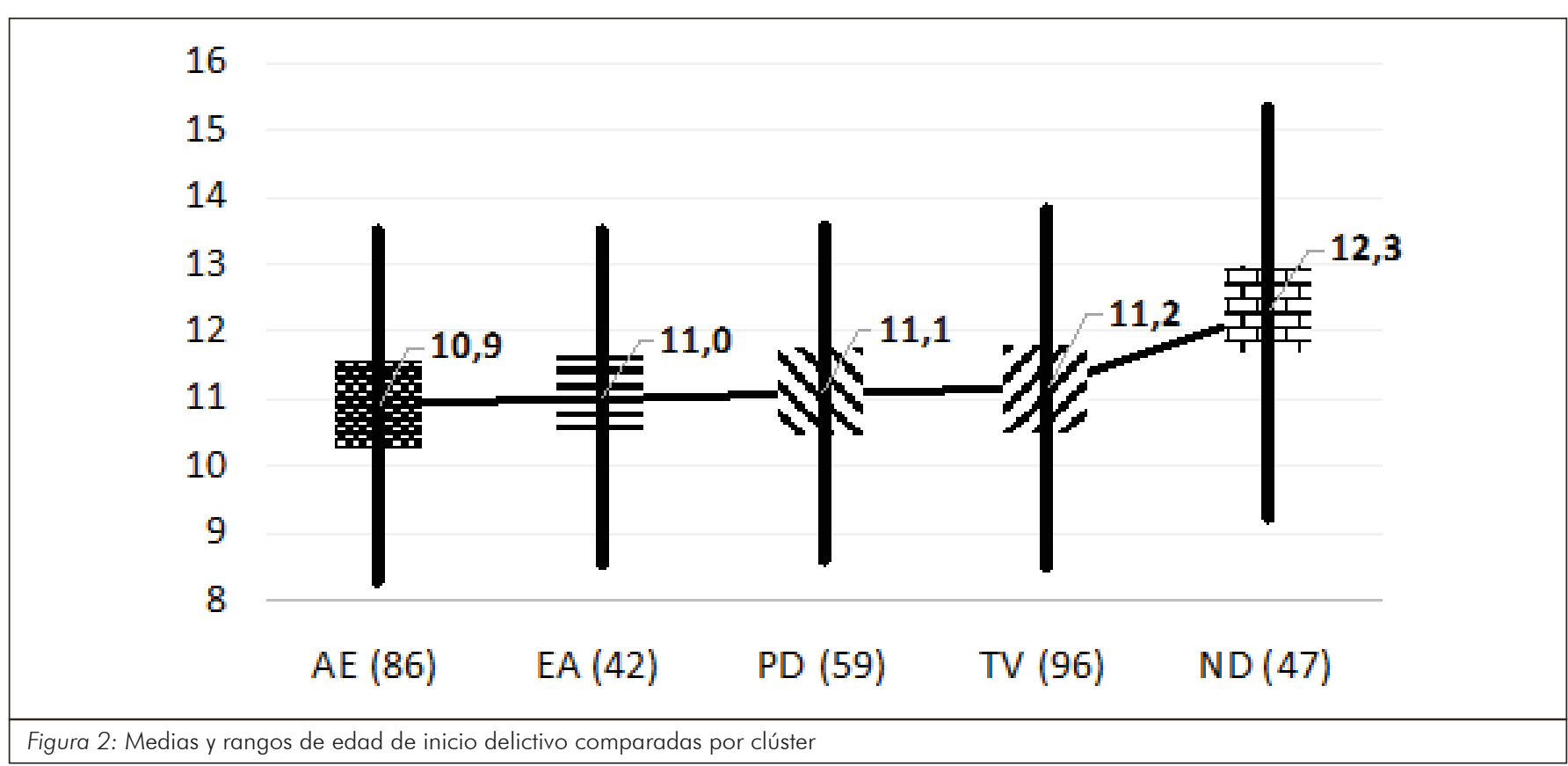

Al observar el comportamiento delictivo a través del tiempo, usando las medianas de los grupos, dada la heterocedasticidad de las distribuciones, se ve como el volumen total de delitos autorreportados difiere significativamente entre clúster ( $\mathrm{KW}=35.7$; $\mathrm{p}$ $=.000$ ), por la distancia entre ND y los otros, aunque con tamaños de efecto pequeños $(G=$ .38 a .48); gráficamente se observa la diferente evolución delictiva, detectando relación directa entre volumen de delitos y compromiso de variables personales (ver figuras 3). A su vez, la frecuencia de delitos violentos (ver figura 4) muestra un volumen significativamente mayor en $\mathrm{EA}$ y $\mathrm{AE}$ al compararlo con ND (KW = 38.8; $\mathrm{p}=$.000), con tamaños de efecto pequeño y moderado ( $G=.24$ a .53 ).

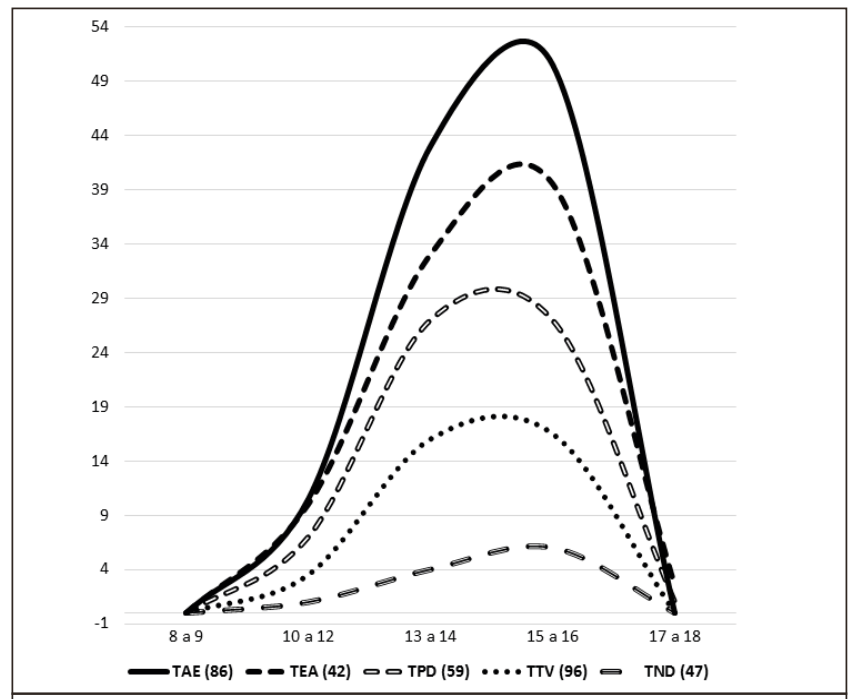

Figura 3: Evolución del volumen de delitos autoreportados por clúster, medidos con EDA
En este plano, la valoración del enganche delictivo promedio de cada clúster, vuelve a ser consistente con la gradiente de variables personales ya referida (ver figura 5), mostrando diferencias estadísticamente significativas entre los grupos ( $\mathrm{KW}=17.8 ; \mathrm{p}=.001$ ) con diferencias en 8 de las 10 comparaciones (sólo no difiere PD con EA y TV), y tamaños de efecto desde pequeños a grandes ( $G=.20$ a .80 ).

El análisis de factores de riesgo criminógenos contextuales muestra diferencias en riesgos totales ( $\mathrm{KW}=34.7 ; \mathrm{p}=.000)$, y salvo en impacto de intervenciones previas (sanciones) que sólo ND puntúa menos que los demás con tamaños de efecto pequeños ( $G=$ .35 a .41), en los cinco factores restantes hay diferencias significativas entre clúster, siendo los

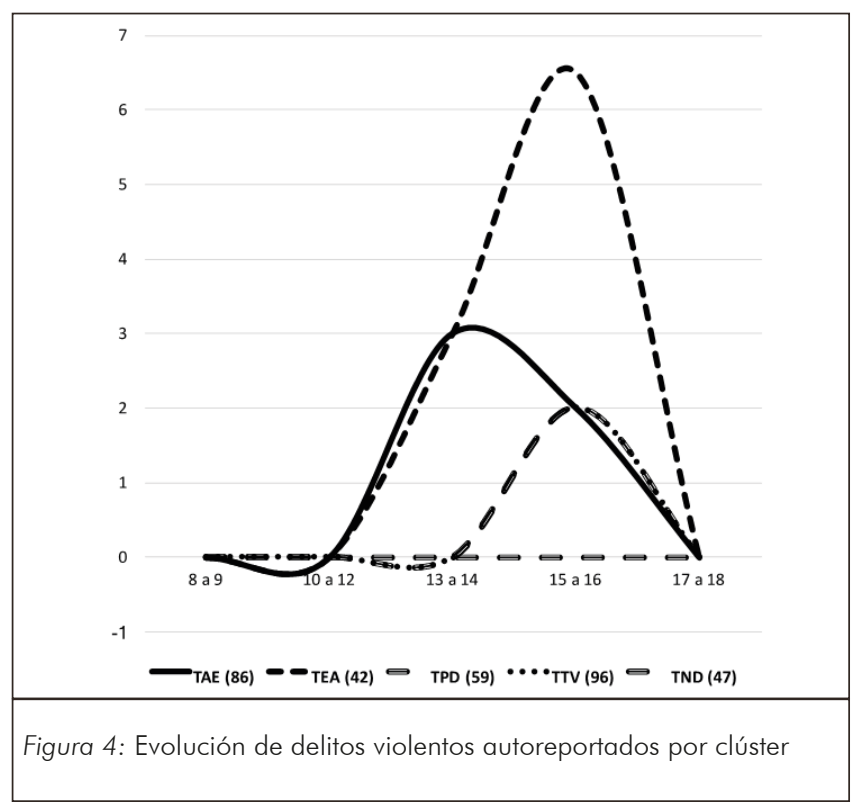



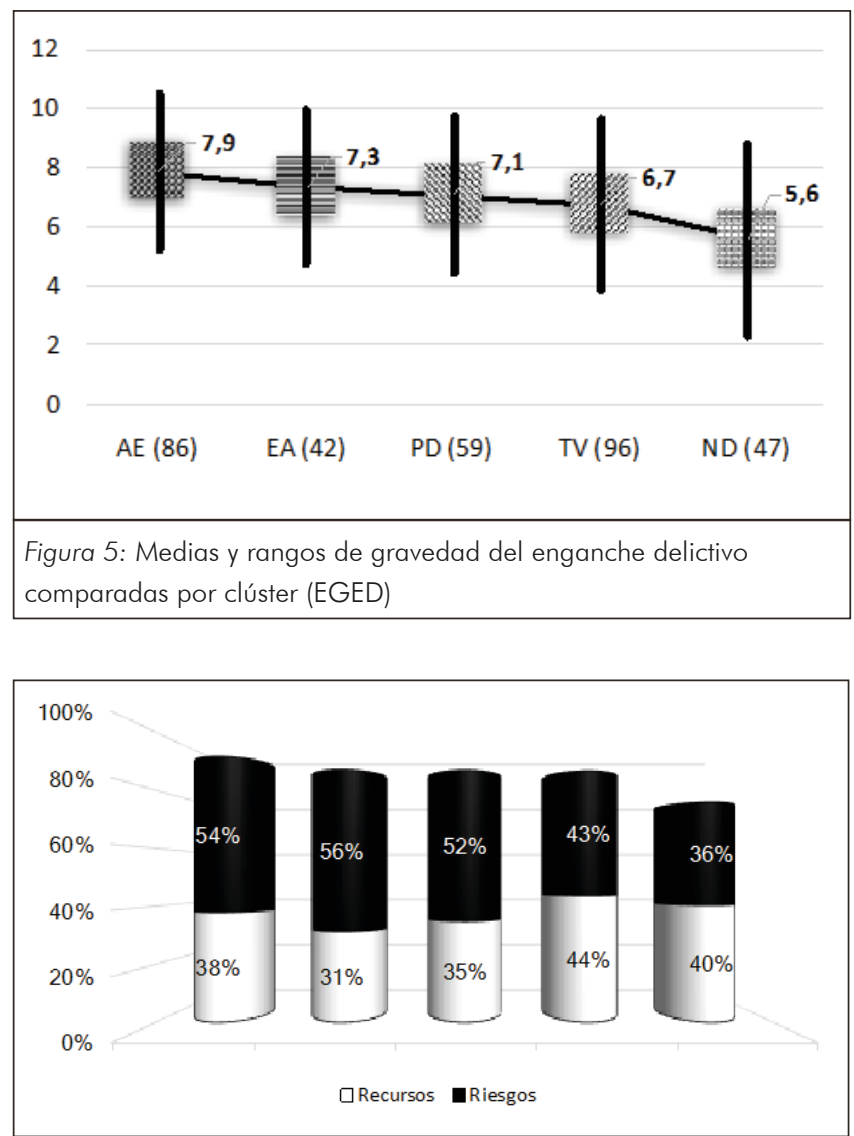

Figura 7: Balance de riesgos y recursos dentro de los clúster

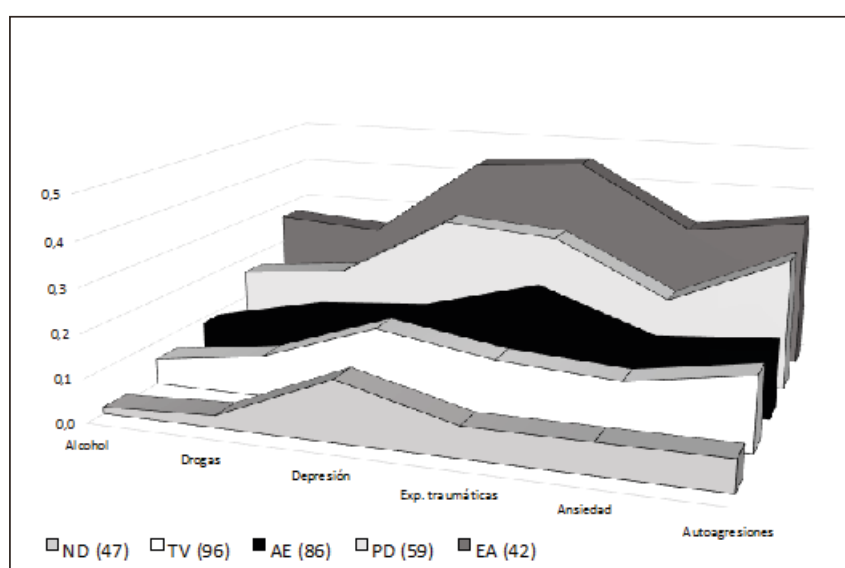

Figura 9: Perfiles de tamizaje de salud mental por clúster, medidos con CESMA

pares con conducta delictiva el riesgo de mayor peso en todas y de menor diferencia entre ellas (KW $=10 ; \mathrm{p}=.040)$. La acumulación de factores de riesgos criminógenos dinámicos se concentran en los clúster $A E, E A$ y $P D$, en contraste con TV y ND que puntúan más bajo (ver figura 6) con tamaños de efecto pequeños y moderados. El grupo de EA, explosivos autodestructivos, muestra diferencias con tamaño de efecto grande en desenganche escolar $(G=.88)$ y drogas $(G=.81)$.

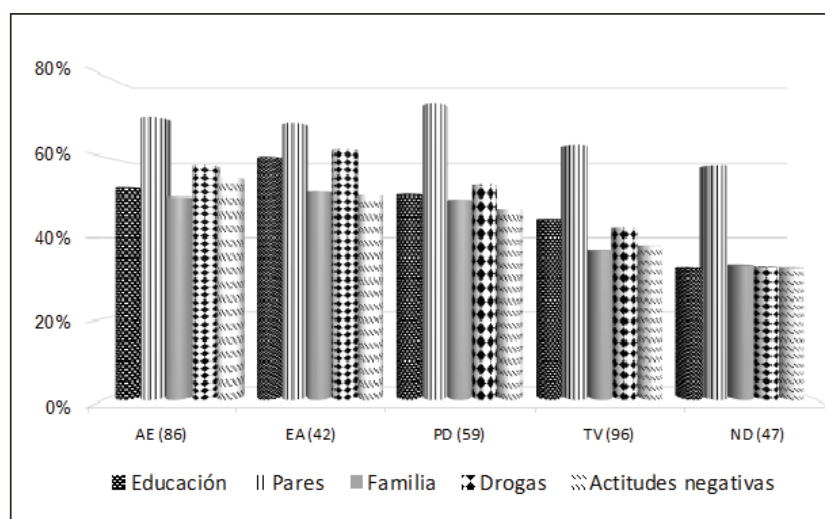

Figura 6: Proporción de riesgos criminógenos promedio entre clúster, medidos con FER-R

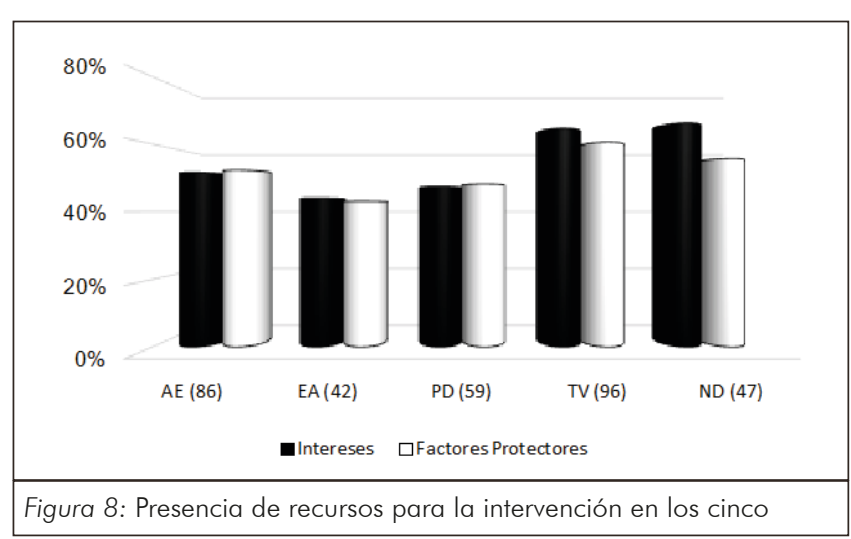

Los recursos para la intervención también difieren significativamente entre clúster (KW $=23.3 ; p=.000)$ en 7 de los 10 contrastes realizados y con tamaños de efecto pequeños y moderados $(G=.31$ a .77$)$, mostrando más recursos en los clúster donde las variables personales se encuentran mas ajustadas, TV y ND (ver figura 7). El análisis interno de recursos revela que el balance positivo de TV y ND se debe a mayor presencia de intereses (ver figura 8), siendo éste un factor determinante para el futuro desistimiento delictivo.

Finalmente, el análisis de tamizaje de salud mental muestra diferencias estadísticamete significativas en los 6 factores de síntomas estudiados $(F=19.3$ a $46.1 ; p=.000$ a .001$)$ con diferencias considerables en 50 de las 60 comparaciones realizadas ( $G=.25$ a 1.37) entre clúster (ver tabla 4). Los perfiles de salud mental detectados (ver figura 9) muestran menor afectación en ND y TV, las dos agrupaciones que poseen menor compromiso de variables personales; las otras tres se reordenan, siendo AE la que menos sintomatología expresa, pues los indicadores de alteración en salud mental están más acentuados en EA y PD. 


\begin{tabular}{|c|c|c|c|c|c|c|c|c|c|}
\hline \multicolumn{10}{|c|}{$\begin{array}{c}\text { Tabla } 4 \\
\text { Comparación de medias de salud mental entre clúster }\end{array}$} \\
\hline & \multirow{2}{*}{$\begin{array}{c}\text { Variables y } \\
\text { Clúster }\end{array}$} & \multicolumn{5}{|c|}{ Índice G de Hedges } & \multicolumn{3}{|c|}{ ANOVA } \\
\hline & & \multicolumn{2}{|c|}{$\mathrm{AE}$} & $\mathrm{EA}$ & PD & TV & gl total & $F$ & $p$ \\
\hline \multirow{24}{*}{$\begin{array}{c}\text { Escalas } \\
\mathrm{MACl}\end{array}$} & \multirow{4}{*}{$\begin{array}{l}\text { Consumo de } \\
\text { alcohol }\end{array}$} & EA & .75 & & & & \multirow{4}{*}{324} & \multirow{4}{*}{43.4} & \multirow{4}{*}{.000} \\
\hline & & PD & .37 & .38 & & & & & \\
\hline & & TV & .15 & .95 & .55 & & & & \\
\hline & & ND & .45 & 1.14 & .84 & .33 & & & \\
\hline & \multirow{4}{*}{$\begin{array}{l}\text { Consumo de } \\
\text { drogas }\end{array}$} & EA & .37 & & & & \multirow{4}{*}{323} & \multirow{4}{*}{19.3} & \multirow{4}{*}{.001} \\
\hline & & PD & .17 & .18 & & & & & \\
\hline & & TV & .18 & .54 & .34 & & & & \\
\hline & & ND & .53 & .89 & .65 & \multirow[t]{2}{*}{.32} & & & \\
\hline & \multirow{4}{*}{ Depresión } & EA & 1.09 & & & & \multirow{4}{*}{324} & \multirow{4}{*}{46.1} & \multirow{4}{*}{.000} \\
\hline & & PD & .70 & .35 & & & & & \\
\hline & & TV & .09 & .92 & .56 & & & & \\
\hline & & ND & .11 & 1.11 & .77 & .19 & & & \\
\hline & \multirow{4}{*}{$\begin{array}{l}\text { Experiencias } \\
\text { traumáticas }\end{array}$} & EA & .68 & & & & \multirow{4}{*}{323} & \multirow{4}{*}{44.0} & \multirow{4}{*}{.000} \\
\hline & & PD & .26 & .40 & & & & & \\
\hline & & TV & .33 & 1.11 & .63 & & & & \\
\hline & & ND & .61 & 1.37 & .91 & .34 & & & \\
\hline & \multirow{4}{*}{ Ansiedad } & EA & .70 & & & & \multirow{4}{*}{322} & \multirow{4}{*}{22.5} & \multirow{4}{*}{.000} \\
\hline & & PD & .49 & .43 & & & & & \\
\hline & & TV & .05 & .67 & .25 & & & & \\
\hline & & ND & .31 & .99 & .57 & .25 & & & \\
\hline & \multirow{4}{*}{ Autoagresiones } & EA & .66 & & & & \multirow{4}{*}{322} & \multirow{4}{*}{36.6} & \multirow{4}{*}{.000} \\
\hline & & PD & .50 & .13 & & & & & \\
\hline & & TV & .04 & .57 & .43 & & & & \\
\hline & & ND & .38 & 1.12 & .89 & .37 & & & \\
\hline
\end{tabular}

\section{DISCUSIÓN}

Los resultados obtenidos permiten sumar evidencia sobre la importancia de las variables de personalidad en la gravedad del enganche delictivo y la persistencia en comportamiento antisocial de los adolescentes, (Wenger \& AndrésPueyo, 2016). En el grupo estudiado las variables de personalidad diferencian cinco agrupaciones en delincuencia persistente adolescente: Antisocial Estabilizado "AE", Explosivo Autodestructivo "EA", Pasivo Desesperanzado "PD", Transgresor Vinculado "TV", y Normal Desviado "ND". En las tres primeras, las características de personalidad jugarían un rol protagónico en la interpretación de las experiencias, interviniendo en el inicio persistencia y agravamiento del comportamiento delictivo, coincidiendo con las propuestas de Moffitt (1993), Piquero et al., (2010), y Fréchette \& Le Blanc (1998).

En el clúster $A E$ domina un estilo antisocial para afrontar los desafios ambientales, con mayor insensibilidad social y la puntuación más elevada en la escala de psicopatía, apoyando la evidencia a favor de esta dimensión psicológica (Frick \& Dickens, 2006; Cooke \& Michie, 2001 ; Pérez et al., 2016) como un factor de riesgo que agrava las conductas antisociales, ya que no interrumpe la acumulación de experiencias criminogénicas. Estas caracteristicas concuerdan con la descripción de Stefurak et al. (2004) de Antisociales Disruptivos, los de predominio Psicopáticos de Taylor et al. (2006) y Estructurados Autónomos de Fréchette y Le Blanc (1998). 
Entre las variables psicológicas diferenciadoras de mayor tamaño de efecto en la literatura aparece la Impulsividad. Los resultados concuerdan con esta dimensión y reconocen al clúster $E A$, con predominio de comportamientos externalizantes, siendo las variables emocionales un factor de riesgo en esta agrupación (Bergheul \& Collete, 2013; Higgins, et al. 2013), lo que se confirma con el predominio de delitos agresivos; por otra parte, se rescata como recurso la probable respuesta diferenciada a las intervenciones, mostrando la potencialidad de vinculación como un recurso que puede sumarse para interrumpir su avance delictivo.

En el clúster PD, la pasividad y desesperanza son características centrales, con escasos recursos personales para afrontar el entorno; los jóvenes se muestran especialmente desmotivados, presentan un patrón delictivo rutinario y repetitivo y sus recursos cognitivos y sociales son escasos, aumentando el riesgo de reincidencia delictiva en contextos criminógenos. Este grupo presenta muchos delitos, pero de baja gravedad y las características personales les pone en una condición de menor receptividad a las intervenciones que buscan cambios en sus conductas.

Lo referido para los primeros tres clústers, identificados suma evidencia para afirmar un compromiso más alto de variables personales como riesgo en la persistencia delictiva, coexistiendo éstas con mayor acumulación de necesidades criminogénicas. Estos resultados coinciden también con los estudios de Alarcón et al. (2005), Fréchette y Le Blanc (1998), Stefurak, et al. (2004) y Taylor et al. (2006), y constituyen hallazgos de consideración para orientar la intervención y ajustar los programas especializados con adolescentes que se inician o mantienen en un actuar antisocial.

Por otro lado, los Clúster TV y ND aparecen con un bajo compromiso de variables personales asociadas al actuar antisocial persistente. El TV, si bien muestra un estilo antisocial en la personalidad, conserva la capacidad de establecer relaciones interpersonales y vincularse afectivamente. Puede ser comprendido como la representación social de "Robin Hood", pues sus delitos se dirigen hacia grupos que se consideran diferentes a su grupo de pertenencia; por ejemplo, sustentaría su actuar antisocial en cogniciones como "sólo le robo a los ricos." Este grupo requiere ser estudiado más en profundidad para reconocer en el entorno factores de riesgo de mayor peso para su persistencia delictiva, pues no aparece igualmente descrito en la literatura científica.

Los adolescentes agrupados en el Clúster ND se asocian con un funcionamiento ajustado, adaptado al medio escolar y a la comunidad; no presentan un perfil de características psicológicas que constituya en si un factor de riesgo, excepto por la falta de poder en las relaciones interpersonales y mayor interés por conformarse al grupo de pares y aceptar sus reglas. El Clúster ND coincide con los reportes de Taylor et al. (2006) en California, con Fréchette y Le Blanc (1998) en Montreal y con Alarcón et al. (2005).

Finalmente, para estas últimas agrupaciones, TV y ND, se infiere que los contextos sociales de su adolescencia han favorecido los riesgos criminógenos, potenciando la aparición del actuar delictivo, pues la variables de personalidad, intereses y recursos no han resultado suficientes a la hora de frenar los comportamientos antisociales, aunque si reducen su gravedad, violencia y peligrosidad. Estos hallazgos resultan de importancia ya que permitirían diferenciar intensidades de intervención, complementando las evaluaciones de riesgos con la receptividad de las variables personales al orientar los programas de tratamiento especializados; estos grupos requerirían de programas de menor intensidad apoyando los modelos RNR y MIID. Además dada sus variables personales, de mayores intereses y recursos, podrían responder muy bien a la relación de ayuda, estrategia de intervención de uso frecuente en los programas con adolescentes que inician su actuar antisocial. A partir de la evidencia reportada en este estudio se formula el Modelo Multidimensional de Intervención Diferenciada con Adolescentes "MMIDA" (Pérez-Luco et al. 2014)

En síntesis, se propone continuar realizando seguimiento a estas agrupaciones y evaluar el impacto de las intervenciones sobre la persistencia delictiva mediante estudios de 
seguimiento. Ello es nuestra principal limitación, al tratarse de un estudio de carácter transversal.

\section{- Financiación}

Los datos de investigación fueron recogidos en el marco del Proyecto I+D+I, FONDEF D08i- 1205 (2010-2014), financiado por CONICYT Chile en colaboración con las instituciones responsable de la administración de justicia juvenil en el país.

\section{- Conflicto de intereses}

Los autores declaran no tener ningún conflicto de intereses.

\section{REFERENCIAS}

Alarcón, P., Pérez-Luco, R., Chesta, S., \& Wenger, L. (2017). Examinando factores de riesgo y recursos para la intervención con adolescentes infractores. En C. Bringas \& M. Novo (Eds.), Psicología Jurídica, conocimiento y práctica. (pp. 431 -448). Sevilla: Universidad de Sevilla. Alarcón, P., Pérez-Luco, R., \& Wenger, L. (2017). Variables personales como riesgo - recurso adaptativo en adolescentes con delincuencia persistente. En M. J. Vásquez \& L. Redondo (Eds.), X Congreso (Inter) Nacional de Psicología Jurídica y Forense. Libro de Resúmenes (pp. 148-152). Sevilla: Universidad de Vigo.

Alarcón, P., Vinet, E., \& Salvo, S. (2005). Estilos de personalidad como riesgo en Desadaptación social. Revista Psyche, 14(1), 3-16.

Alarcón, P., Wenger, L., Chesta, S., \& Salvo, S. (2012). Validez predictiva del instrumento Evaluación de Riesgos y Recursos (FER-R) para la intervención en adolescentes chilenos infractores de ley: estudio preliminar. Universitas Psychologica, $17(4), 1183-1195$.

Andrews, D., Bonta, J., \& Wormith, S. (2011). The risk-need-responsivity model (RNR) Model. Does adding the Good Lives Model contribute to effective crime prevention? Criminal Justice and Behavior, 38(7), 735-755. https://doi. org/10.1177/0093854811406356

Assink, M., van der Put, C. E., Hoeve, M., de Vries, S. L. A., Stams, G. J. J. M., \& Oort, F. J. (2015). Risk factors for persistent delinquent behavior among juveniles: A meta-analytic review. Clinical Psychology Review, 42, 47-61. http:// doi.org/10.1016/i.cpr.2015.08.002
Barlett, C. P., \& Anderson, C. A. (2012). Direct and indirect relations between the big 5 personality traits and aggressive and violent behavior. Personality and Individual Differences, 52(8), 870-875. paid.2012.01.029

Bergheul, S., \& Collette, J. (2013). Personality traits predictive of delinquency: A comparative study between a population of young offenders and conventional Canadians girls in New Brunswick. Anales Medico-Psychologiques, 171, 220-225. http://doi.org/10.1016/i.amp.2012.06.028

Berríos, C., Chesta, S., Wenger, L., Lagos, L., Alarcón, P., \& Pérez-Luco, R. (2014). Cuestionario de exploración de salud mental para adolescentes "CESMA". Instrumento del protocolo de evaluación diferenciada MMIDA. Temuco, Chile: Universidad de La Frontera.

Bosick, S. J., Bersani, B. E., \& Farrington, D. P. (2015). Relating clusters of adolescent problems to adult criminal trajectories: a personcentered, prospective approach. Journal of Developmental and Life-Course Criminology, 1(2), 169-188. http://doi.org/10.1007/ s40865-015-0009-y

Castañeda, A., Garrido-Fernández, M., \& Lanzarote, M. (2012). Juvenile offenders who assault their parents: A study of personality traits and parenting styles. Revista de Psicología Social, 27, 157-167. http://doi. org/10.1174/021347412800337933

Cooke, D. J., \& Michie, C. (2001). Refining the construct of psychopathy: towards a hierarchical model. Psychological Assessment, 13(171188).

Cutrín, O., Gómez-Fraguela, J. A., Maneiro, L., \& Sobral, J. (2017). Effects of parenting practices through deviant peers on nonviolent and violent antisocial behaviours in middleand late-adolescence. The European Journal of Psychology Applied to Legal Context, 9(2), 75-82. http://doi.org/10.1016/i. ejpal.2017.02.001

De Bolle, M., Beyers, W., De Clercq, B., \& De Fruyt, F. (2012). General personality and psychopathology in referred and nonreferred children and adolescents: An investigation of continuity, pathoplasty, and complication 
models. Journal of Abnormal Psychology, 121(4), 958-970. http://doi.org/10.1037/ a0027742

Fariña, F., Vázquez, M. J., \& Arce, R. (2014). ¿Está mediada la gravedad delictiva y cronicidad de los delincuentes juveniles por la competencia cognitivo-comportamental? Universitas Psychologica, 13(2), 15-27. http:// doi.org/10.11144/Javeriana.UPSY13-3

Farrington, D. P., Ttofi, M. M., \& Piquero, A. R. (2016). Risk, promotive, and protective factors in youth offending: Results from the Cambridge study in delinquent development. Journal of Criminal Justice, 45, 63-70. http://doi. org/10.1016/i.jcrimius.2016.02.014

Faúndez, X., \& Vinet, E. (2009). Tipología Empírica de Adolescentes Consumidores de Drogas según el MACl. Psykhe, 18(2), 19-35. http://doi.org/10.4067/s071822282009000200002

Ferrer, L., \& Kirchner, T. (2014). Suicidal tendency in a sample of adolescent outpatients with adjustment disorder: gender differences. Comprehensive Psychiatry, 55(6), 1342-1349. https://doi.org/10.1016/i. comppsych.2014.04.017

Forth, A. E., Kosson, D. S., \& Hare, R. D. (2003). The Psychopathy Checklist: Youth Version. Toronto, Ontario: Multi-Health Systems.

Fréchette, M., \& Le Blanc, M. (1998). Délinquances et délinquants (8 ed.). Montreal, Québec, Canadá: Gaêtan Morin.

Frick, P. J., \& Dickens, C. (2006). Current perspectives on conduct disorder. Current Psychiatry Reports, 8, 59-72. https://doi. org/10.1007/s $11920-006-0082-3$

Gabriel, K. R. (2002). Goodness of fit of biplots and correspondence analysis. Biometrika, 89(2), 423-436. https://doi.org/10.1093/ biomet/89.2.423

García, C. H., Moral, J., Frías, M., Valdivia, J., \& Díaz, H. (2012). Factores de riesgo familiares y sociodemográficos para la psicopatía en un grupo de prisioneros. European Journal of Psychology Applied to Legal Context, 4(2), 119-134.

Glowacz, F., \& Born, M. (2013). Do adolescent child abusers, peer abusers, and non-sex offenders have different personality profiles? European Child \& Adolescent Psychiatry, 22(2), 1 17-125. doi: 10.1007/s00787-012-0333-2 Gómez, B. M., Arango, T. E., Molina, D., \& Barceló, E. (2010). Características de la teoría en el trastorno disocial de la conducta. Psicología desde El Caribe, 26, 103-118.

Guillén, N., Roth, E., Alfaro, A., \& Fernández, E. (2015). Youth alcohol drinking behavior: Associated risk and protective factors. Revista Iberoamericana de Psicología y Salud, 6(2), 53-63. http://doi.org/10.1016/i. rips.2015.03.001

Hare, R. D. (2003). Manual for the Hare Psychopathy Checklist- Revised (2a ed.). Toronto, Ontario, Canadá: Multi-Health Systems.

Higgins, G., Kirchner, E., Ricketts, M., \& Marcum, C. (2013). Impulsivity and Offending from Childhood to Young Adulthood in the United States: A Developmental Trajectory Analysis. International Journal of Criminal Justice Sciences (IJCJS), 8(2), 182-197.

Jolliffe, D., \& Farrington, D. P. (2009). A systematic review of the relationship between childhood impulsiveness and later violence. In M. McMurran \& R. Howard (Eds.), Personality, personality disorder and violence (pp. 40-61). London, UK: Wiley.

Jones, S., Miller, J., \& Lynam, D. (2011). Personality, antisocial behavior, and aggression: A meta-analytic review. Journal of Criminal Justice, 39(4), 329-337. http://doi. org/10.1016/i.jcrimius.2011.03.004

Kennedy, T., Burnett, K., \& Edmonds, W. (2011). Intellectual, behavioral, and personality correlates of violent vs. non-violent juvenile offenders. Aggressive Behavior, 37(4), 315 325. http://doi.org/10.1002/ab.20393

Lagos, L., Pérez-Luco, R., Chesta, S., \& Wenger, L. (2014). Escala de gravedad del enganche delictivo "EGED". Instrumento del protocolo de evaluación diferenciada MMIDA. Temuco, Chile: Universidad de La Frontera.

Le Blanc, M., \& Loeber, R. (1998). Developmental criminology updated. Crime and Justice, 23, 115-198. http://doi.org/10.1086/449270

Le Corff, Y., \& Toupin, J. (2009). Comparing 
persistent juvenile delinquents and normative peers with the Five-Factor Model of Personality. Journal of Research in Personality, 43(6), 1105-1108. http://doi.org/10.1016/i. irp.2009.06.011

Le Corff, Y., \& Toupin, J. (2010). The FiveFactor Model of personality at the facet level: Association with antisocial personality disorder symptoms and prediction of antisocial behavior. Journal of Psychopathology and Behavioral Assessment, 32(4), 586-594. http://doi. org/10.1007/s10862-010-9180-y

Lenhard, W. \& Lenhard, A. (2016). Calculation of effect sizes. Available: Recuperado de https:// www.psychometrica.de/effect size.html.

Llorca-Mestre, A., Malonda-Vidal, E., \& SamperGarcía, P. (2017). Prosocial reasoning and emotions in young offenders and nonoffenders. The European Journal of Psychology Applied to Legal Context, 9(2), 65-73. http:// doi.org/10.1016/i.eipal.2017.01.001

Loeber, R., Farrington, D. P., Stouthamer-Loeber, M., Moffitt, T. E., Caspi, A., White, H. R., ... Beyers, J. M. (2003). The development of male offending: Key findings from fourteen years of the Pittsburgh Youth Study. In T. Thornberry \& M. Krohn (Eds.), Taking stock of delinquency: An overview of findings from contemporary longitudinal studies (pp. 93-136). New York, NY: Kluwer-Plenum.

Martins-Vairinhos, V., \& Galindo, M. P. (2004). Biplots PMD - data mining centrada em Biplots. Apresentação de um protótipo. In XI Jornadas de Classificação e Análise de Dados (pp. 1-18). Lisboa: Associação Portuguesa de Classificação e Analise de Dados.

Millon, T. (1990). Toward a new personology: An evolutionary model. New York, NY: Wiley.

Millon, T. (1993). Manual of Millon Adolescent Clinical Inventory. Minneapolis, MN: National Computer Systems.

Millon, T., \& Davis, R. (1998). Trastornos de la personalidad: Más allá del DSM-IV. Barcelona, España: Masson.

Millon, T., Simonsen, E., Birket-Smith, M., \& Davis, R. (2003). Psychopathy: Antisocial criminal and violent behaviour. New York: Guilford Press.

Moffitt, T. E. (1993). Adolescence-limited and life-course-persistent antisocial behavior: A developmental taxonomy. Psychological Review, 100(4), 674-701.

Moffitt, T. E. (2003). Life course-persistent and adolecence-limited antisocial behavior: A 10year research review and research agenda. In B. Lahey, T.-E. Moffitt, \& A. Caspi (Eds.), Causes of conduct disorder and juvenile delinquency (pp. 49-75). NY: Guilford Press.

Moffitt, T. E. (2015). Life-course-persistent versus adolescence-limited antisocial behavior. In D. Cicchetti \& D. J. Cohen (Eds.), Developmental Psychopathology (Second Edition). Hoboken, NJ, USA: John Wiley \& Sons, Inc. http://doi. org/10.1002/9780470939406.ch15

Moffitt, T. E., Arseneault, L., Belsky, D., Dickson, N., Hancox, R. J., Harrington, H., ... Caspi, A. (2011). A gradient of childhood self-control predicts health, wealth, and public safety. Proceedings of the National Academy of Sciences of the United States of America, 108(7), 2693-2698. http://doi. org/10.1073/pnas. 1010076108

Morizot, J. (2015). The contribution of temperament and personality traits to criminal and antisocial behavior development and desistance. In J. Morizot \& L. Kazemian (Eds.), The Development of Criminal and Antisocial Behavior (pp. 137-165). Switzerland: Springer International Publishing. http://doi. org/10.1007/978-3-319-08720-7

Morrell, L., \& Burton, D. (2014). An Exploration of Psychopathy in Self-Report Measures Among Juvenile Sex Offenders. International Journal of Ofender Therapy and Comparative Criminology, 58(5), 550-566. https://doi. org/10.1177/0306624X13479186

Murrie, D., \& Cornell, D. (2000). The Millon adolescent clinical inventory and psychopathy. Journal of Personality Assessment, 75(1), 110-125. http://doi.org/10.1207/ S15327752JPA7501 8

Olver, M. E., Stockdale, K. C., \& Wormith, J. S. (2014). Thirty years of research on the level of service scales: A meta-analytic examination of predictive accuracy and sources of variability. Psychological Assessment, 26(1), 156-176. http://doi.org/10.1037/a0035080 
Pérez, B., Herrero, J., Velasco, J., \& RodríguezDíaz, F. J. (2015). A contrastive analysis of the factorial structure of the PCL-R: Which model fits best the data? European Journal of Psychology Applied to Legal Context, 7(1), 23-30. http://dx.doi.org/10.1016/i. ejpal2014.10.001

Pérez, B., Rodríguez-Díaz, F. J., Herrero, J., \& Fernández-Suárez, A. (2016). Perfil del psicópata institucionalizado sin comportamiento criminal previo a su entrada en prisión. Terapia Psicológica, 34(2), 81-91.

Pérez-Luco, R., Alarcón, P., Zambrano, A., Alarcón, M., Chesta, S., \& Wenger, L. (2017). Taxonomía de la delincuencia adolescente con base en evidencia chilena. En C. Bringas \& $M$. Novo (Eds.), Psicología Jurídica, conocimiento y práctica. (pp. 251-270). Sevilla: Universidad de Sevilla.

Pérez-Luco, R., Lagos, L., Chesta, S., \& Báez, C. (2014). Escala de delincuencia autorevelada "EDA". Instrumento del protocolo de evaluación diferenciada MMIDA. Temuco, Chile: Universidad de La Frontera.

Pérez-Luco, R., Zambrano, A., Alarcón, P., \& Alarcón, M. (2014). Informe final Proyecto D08i-1205: "Estrategia ecosistémica especializada de intervención diferenciada para favorecer la integración psicosocial de adolescentes infractores de ley". Santiago de Chile: FONDEF-Universidad de La Frontera.

Piquero, A., Farrington, D. P., Nagin, D. S., \& Moffitt, T. E. (2010). Trajectories of offending and their relation to life failure in late middle age: Findings from the Cambridge study in delinquent development. Journal of Research in CrimeandDelinquency, 47(2), 151 - 173.http:// doi.org/10.1177/0022427809357713

Piquero, A. R., Jennings, W. G., \& Barnes, J. C. (2012). Violence in criminal careers: A review of the literature from a developmental lifecourse perspective. Aggression and Violent Behavior, 17(3), 171-179. https://doi. org/10.1016/i.avb.2012.02.008

Rodríguez-Díaz, F. J., Bringas, C., Rodríguez, L., López-Cepero, J., Pérez, B., \& Estrada, C. (2011). Drug abuse and criminal family records in the criminal history of prisoners.
European Journal of Psychology Applied to Legal Context, 3(2), 89-105.

Romm, S., Bockian, N., \& Harvey, M. (1999). Factor-based prototypes of the Million Adolescent Clinical Inventory in adolescents referred in residential treatment. Journal of PersonalityAssessment, 72(1), 125-143.http:// doi.org/10.1207/s15327752ipa7201_8

Salekin, R. T., Ziegler, T., Larrea, M., Anthony, V., \& Bennett, A. (2003). Predicting dangerousness with two Millon adolescent clinical inventory psychopathy scales: the importance of egocentric and callous traits. Journal of Personality Assessment, 80, 154-163.

Siödin, A.-K., Wallinius, M., Billstedt, E., Hofvander, B., \& Nilsson, T. (2017). Dating violence compared to other types of violence: similar offenders but different victims. The European Journal of Psychology Applied to Legal Context, 9(2), 83-91. http://doi. org/10.1016/i.ejpal.2017.03.001

Stefurak, T., \& Calhoun, G. (2007). Subtypes of female juvenile offenders: a cluster analysis of the Millon Adolescent Clinical Inventory. International Journal of Law and Psychiatry, 30(2), 95-111. http://doi.org/10.1016/i. iilp.2006.04.003

Stefurak, T., Calhoun, G. B., \& Glaser, B. A. (2004). Personality typologies of male juvenile offenders using a cluster analysis of the Millon Adolescent Clinical Inventory introduction. International Journal of Offender Therapy and Comparative Criminology, 48(1), 96-110. http://doi. org/10.1177/0306624X03258478

Taylor, J., Kemper, T. S., Loney, B. R., \& Kistner, J. a. (2006). Classification of severe male juvenile offenders using the $\mathrm{MACl}$ clinical and personality scales. Journal of Clinical Child and Adolescent Psychology, 35(1), 90-102. http:// doi.org/10.1207/s 15374424jiccp3501_8

Tremblay, R. E., Côté, S. M., Salla, J., \& Michel, G. (2017). The development of aggression from early childhood to adulthood. En P. Sturmey (Ed.), The wiley handbook of violence and aggression (pp. 1-15). New York, NY: Wiley. http://doi.org/10.1002/9781119057574. whbva004 
Vinet, E., \& Forns, M. (2008). Normas Chilenas para el $\mathrm{MACl}$ : Una integración de criterios categoriales y dimensionales. Terapia Psicologica, 26(2), 151-163.

Wareham, J., Dembo, R., Poythress, N., Childs, K., \& Schmeidler, J. (2009). A Latent Class Factor Approach to Identifying Subtypes of Juvenile Diversion Youths Based on Psychopathic Features. Behavioral Sciences and the Law, 27, 71-95. http://doi.org/10.1002/bsl.844
Wenger, L., \& Andres-Pueyo, A. (2016). Tests personológicos y clínicos en Español para evaluar adolescentes infractores. Papeles del Psicologo, 37(2), 89-106.

Zúñiga, D., Vinet, E., \& León, E. (2011). Caracterización psicométrica del Psychopathy Checklist: Youth Version (PCL:YV) en adolescentes Chilenos. Terapia Psicologica, 29(1), 25-31. http://doi.org/10.4067/ S0718-48082011000100003 